\title{
Mathematical modeling of drug release from swellable polymeric nanoparticles
}

\author{
Soha Azadi ${ }^{1,2}$, Hajar Ashrafi ${ }^{1,2}$, Amir Azadi ${ }^{1,2^{*}}$ \\ ${ }^{1}$ Pharmaceutical Sciences Research Center, Shiraz University of Medical Sciences, Shiraz, Iran. \\ ${ }^{2}$ Department of Pharmaceutics, School of Pharmacy, Shiraz University of Medical Sciences, Shiraz, Iran.
}

\begin{tabular}{|c|c|}
\hline ARTICLE INFO & ABSTRACT \\
\hline Article history: & \multirow{10}{*}{$\begin{array}{l}\text { This study aims to provide a comparative mathematical analysis of drug release from swellable polymeric } \\
\text { delivery systems to find a general model applicable to multi-mechanistic release. Drug release data from various } \\
\text { swellable polymeric nanoparticles extracted from the literatures were applied to the eight conventional models. } \\
\text { Coefficient of determination }\left(\mathrm{R}^{2}\right) \text { and absolute percent error (E\%) were calculated for each set as well as the } \\
\text { overall error (OE), the number of error (NE) and the akaike information criterion (AIC) for all sets. The model } \\
\text { has the highest } \mathrm{R}^{2} \text { and the number of the error, as well as both the lowest overall error (OE) and the akaike } \\
\text { information criterion, was considered as the best one. Among the models Weibull (W) model produced } \mathrm{R}^{2} \text { and } \\
\text { OE values of } 0.93 \text { and } 8.79 \text {, respectively. Also, the AIC value and the number of errors less than } 5 \% \text { for the } \\
\text { model was }-34.93 \text { and } 46.15 \% \text { of a total number of data sets respectively. Mathematical modeling of drug } \\
\text { release from a carrier is often attempted to recognize the main determinants of the drug release rate from the } \\
\text { carrier with the final goal of the identification of the ideal set of conditions leading to the desired release profile } \\
\text { in vivo. }\end{array}$} \\
\hline Rece & \\
\hline Ac & \\
\hline /04/2017 & \\
\hline Key words: & \\
\hline Mathematical modeling, drug & \\
\hline release, swellable polymeric & \\
\hline nanoparticles, & \\
\hline & \\
\hline & \\
\hline
\end{tabular}

\section{INTRODUCTION}

In recent decades, increasing attention has been devoted to the nanotechnology role in various sciences including biomedical and pharmaceutical fields. Nanoparticles have emerged as promising carriers for the delivery of a wide range of therapeutic drugs. Currently many materials such as polymers, lipids, proteins, carbons, and inorganic metals are under investigation for drug delivery (Yoon et al., 2013). To improve the delivery systems, several types of nanoparticulate systems including polymeric nanoparticles (Hamidi et al., 2011; Azadi et al., 2012; Hamidi et al., 2012; Azadi et al., 2015), polymeric micelles, solid nanoparticles, lipid-based nanoparticles, such as solid lipid nanoparticles (SLN), nanostructured lipid carriers (NLC) and lipid drug conjugate (LDC) (Ashrafi et al., 2013), nanoliposomes (Azadi and Ashrafi, 2016), inorganic nanoparticles, dendrimers, magnetic nanoparticles, Ferrofluids,

\section{* Corresponding Author}

Amir Azadi, Department of Pharmaceutics, School of Pharmacy, Shiraz University of Medical Sciences, Shiraz, Iran.

E-mail: aazadi@sums.ac.ir and quantum dots have been introduced and investigated previously (Hamidi et al., 2008). Among the various types of nanocarriers, polymeric nanoparticles which are classified to swellable and non-swellable ones, have been considered as promising devices in controlled drug release systems. Thus swellable polymeric nanoparticles, are frequently used in such formulations to improve the therapeutic value of various drugs and bioactive molecules due to their interesting physiochemical properties containing improvement of bioavailability, specificity and prolongation of circulating time, as well as reduction of the drug side effects, and risks of toxicity (Kumari et al., 2010). As a matter of fact "drug release" refers to a process in which a drug molecule migrates from an initial position in a polymeric system to release medium and after subjecting to pharmacokinetic procedures, eventually becoming available for its pharmacological action. Accordingly, the drug release from nanoparticles influences its pharmacological effects as a major determinant. Studies on drug release kinetics provide important information into realizing and optimizing of such formulations (Hamidi et al., 2013). A mathematical modeling of drug release from a carrier is often attempted to recognize the release mechanisms considered as an 
essential determinant for designing of controlled drug release systems. Additionally, kinetics with one or two parameters can represent several release data (Barzegar-Jalali et al., 2008). Kinetic models are also capable of characterizing the in vivo release profile. Therefore, the use of in vitro drug release data to predict in vivo performance of drug substances can be considered as the rational development of controlled release formulations. This study aims to provide a comparative mathematical analysis of drug molecules release from swellable polymeric drug delivery systems to find a general model the most applicable to determine the in vivo profile.

\section{MATERIALS AND METHODS}

To study the release kinetics of various drug molecules from the swellable polymeric nanoparticles, such as chitosan, alginate, gelatin, hyaluronic acid and etc, the release data were extracted from the literatures and fitted to the following eight conventional equations:

\section{Zero order equation:}

$$
F=k_{o} \cdot t
$$

where $\mathrm{F}$ stands for the fraction of drug released up to time $\mathrm{t}$ and $\mathrm{k}_{\mathrm{o}}$ is the zero-order release rate constant

\section{First order equation}

$$
\ln (1-F)=-k_{l} t
$$

where $\mathrm{k}_{1}$ stands for first-order release rate constant

\section{Higuchi's equation}

$$
F=k_{H} \cdot t^{1 / 2}
$$

where $\mathrm{k}_{\mathrm{H}}$ represents the Higuchi release rate constant

\section{Hixson-Crowell model}

$$
1-(1-F)^{1 / 3}=-k_{H C} \cdot t
$$

where $\mathrm{k}_{\mathrm{HC}}$ stands for Hixson-Crowell release rate constant

\section{Square root of mass}

$$
1-(1-F)^{1 / 2}=K_{S R} \cdot t
$$

where $\mathrm{k}_{\mathrm{SR}}$ stands for the square root of mass model release rate constant

\section{Three seconds root of mass:}

$$
-(1-F)^{2 / 3}=K_{T S R} \cdot t
$$

where $\mathrm{k}_{\mathrm{TSR}}$ stands for Three seconds root of mass model release rate constant

\section{Weibull equation}

$$
\ln [-\ln (1-F)]=\beta \ln t d+\beta \ln t
$$

Where td stands for the lag time before the drug release takes place and $\beta$ characterizes the shape of the release curve

\section{Korsmeyer-Peppas model}

$$
\ln F=\ln k_{\mathrm{kp}}+n \ln t
$$

Where $\mathrm{k}_{\mathrm{KP}}$ is a constant corresponding to the geometric and structural characteristics of the device and " $n$ " is the release exponent which determined the mechanism of the drug release. Sum square of errors (SSE), sum square of regression (SSR) and sum square of total variation (SST) were calculated by further equations:

$$
\begin{gathered}
\mathrm{SSE}=\sum_{i=1}^{n} \mathrm{e}^{2}=\sum_{i=1}^{n}(y i-y f i)^{2} \\
\mathrm{SST}=\sum_{i=1}^{n}(\mathrm{yi}-\mathrm{y})^{\wedge} 2 \\
S S R=S S T-S S E \\
R^{2}=1-\frac{S S E}{S S T}
\end{gathered}
$$

Where yi show the vector of dependent observed variables for an observation, yfi is a fitted variable value, $y$ is the mean value of yi and $\mathrm{e}$ is the error vector.

Also, the accuracy and predictability of the models were distinguished by computing of absolute percent error $(\mathrm{E} \%)$ for each set as well as number of error (NE) for all sets as given by Eqs.

$$
\begin{gathered}
\mathrm{E}=\frac{100}{N} \sum_{i=1}^{n} \frac{\mathrm{Fcal} \mathrm{i}-\mathrm{F} \text { obs } \mathrm{i}}{F \text { obs } i} \\
N E(i)=\frac{100 \times n(i)}{N}
\end{gathered}
$$

$\mathrm{F}$ cal $\mathrm{i}$ and $\mathrm{F}$ obs $\mathrm{i}$ denote calculated fraction and an observed fraction of drug released at the $i$ th sample, respectively. The value of $\mathrm{N}$ is the number of data in each set and $\mathrm{n}(\mathrm{i})$ represents the number of data points with the equal or lower "E" than "i" (Azadi et al., 2013)

The Akaike Information Criterion (AIC) is also used to examine the applicability of the release models. The Akaike Information Criterion is a measure of goodness of fit based on maximum likelihood.

$$
A I C=n \times \ln (W S S R)+2 \times p
$$

Where $\mathrm{n}$ is the number of dissolution data points, $\mathrm{p}$ is the number of the parameters of the model, WSSR is the weighed sum of square of residues, calculated by this process:

$$
\text { WSSR } \left.=\sum_{k i=1}^{n}[\text { wi(Fcal i }- \text { Fobs i })^{\wedge} 2\right]
$$

Where $\mathrm{w}$ is an optional weighing factor and Fcal $\mathrm{i}$ and Fobs $\mathrm{i}$ denote calculated fraction and observed a fraction of drug released at the sample, respectively (Costa and Lobo, 2001). 


\section{RESULTS AND DISCUSSION}

The release data of all formulations were fitted to 8 models mentioned above. The overall error, number of errors less than 5,10,15 and $20 \%$ and akaike information criterion for the models are shown in Table $1 . \mathrm{R}^{2}$ is a statistical measure indicates how well data fit a statistical model. But in order to have more reliable results, absolute percent error (E) for each set and number of error (NE) for all sets were also calculated. These parameters represent the accuracy and predictability of models. The model had the highest $\mathrm{R}^{2}$ and the number of the error, as well as the lowest overall error (OE), was considered as the best one. Also, the akaike information criterion was calculated to demonstrate the goodness of data fitting. The model associated with the smallest value of AIC is regarded as giving the best fit out of the models. Among the models, Weibull (W) model produced $\mathrm{R}^{2}$ and $\mathrm{OE}$ values of 0.93 and 8.79 , respectively. Also, the number of errors less than $5 \%$ was $46.15 \%$ of a total number of data set and akaike information criterion of this model is -34.93 (which is the smallest value of all). The values of $\mathrm{E} \%$, AIC, the coefficient of determination $\left(\mathrm{R}^{2}\right)$ and the regression parameters extracted from each data set was fitted to Weibull equation are given in Table 2.

Residual sum square (RSS), total sum square (TSS) and residual minus square (RMS) were calculated to evaluate the coefficient of determination $\left(\mathrm{R}^{2}\right)$ for each model and the accuracy of the best-fitted data by the suggested mathematical models. The values of them which extracted from Weibull equation are shown in Table 3. The relative sizes of the sums of squares terms demonstrate how "good" the regression is in terms of fitting the calibration data. If the regression is "perfect", $\mathrm{R}^{2}$ will be 1 . Weibull is an empirical model has been successfully used in analysis of both rapid and extended release data due to its versatility (Azadi et al., 2013). This study provides valuable evidence for the practicable use of the Weibull model in drug release phenomena from swellable polymeric nanoparticles. Likely there are also several studies have experimentally investigated that, the release data from swellable polymeric nanoparticles fit best with the weibull model (Yang et al., 2000; Adibkia et al., 2007; Aksungur et al., 2011; Ji et al., 2011; Bei et al., 2012; Azadi et al., 2013; Sun et al., 2014; Liu et al., 2015; Ashrafi and Azadi, 2016; Jafari-Aghdam et al., 2016). Among the studied models,
Weibull is regarded as a favorable model which includes parameters that are sensitive to ranges of release profile. This model offers a simple relation between the parameters and geometrical characteristic of system as well as Kosmidis et al. (2003) experimentally indicate the dependence of parameters on the specific surface. As previously mentioned, $t_{d}$ in Weibull equation is a location parameter denotes the lag time before the onset of drug release procedure, while $\beta$, a shape parameter, characterizes the shape of the release curve (Azadi et al., 2013) and it can be linked to physiological effect as it has been discussed in earlier study (vanBoekel, 2002). For more identification, when $\beta=1$, the shape of curve becomes an exponential profile. If $\beta$ has a higher value than 1 , the curve gets sigmoidal form and finally with the $\beta$ lower than 1 the equation provides parabolic model (Kalam et al., 2007). It is obvious that the external condition is capable to influence the release kinetic and be determined by the shape parameter as well (vanBoekel, 2002). Moreover, for better characterization of the drug release mechanisms, KorsmeyerPeppas semi-empirical model was applied. In addition, Korsmeyer-Peppas model can correlate release data with release mechanisms which Weibull model cannot provide. At 1983, Korsmeyer et al. provided a relationship in drug release from a polymeric system. To discover the overall mechanism of drug release, first $60 \%$ drug release data was fitted in KorsmeyerPeppas model (Dash et al., 2010).

$$
\ln \mathrm{F}=\ln \mathrm{k}_{\mathrm{kp}}+\mathrm{n} \ln \mathrm{t}
$$

Where $\mathrm{F}$ stands for the fraction of drug released up to time $t, k_{K P}$ is a Korsmeyer-Peppas release rate constant and " $n$ " is the release exponent. The $n$ value is used to characterize different release mechanisms as given further.

According to the Korsmeyer- Peppas equation (Dash et al., 2010), $0.45 \leq \mathrm{n}$ are described with a Fickian diffusion mechanism, $0.45<\mathrm{n}<0.89$ with non-Fickian transport, $\mathrm{n}=0.89$ with Case II transport, and $\mathrm{n}>0.89$ with super case II transport.

The release exponent of Korsmeyer-Peppas equation for all data sets are shown in Table 4. The data produced the $\mathrm{n}<0.45$, $0.45<\mathrm{n}<0.89$ and $0.98<\mathrm{n}$ percent of $61.5 \%, 33.9 \%$ and $4.6 \%$, respectively. Based on results Weibull model seems to be more applicable for identification of drug release from swellable polymeric nanoparticles leading to the desired release profile in vivo.

Table 1: The overall error, number of errors less than 5, 10, 15 and $20 \%$ and akaike information criterion for the models.

\begin{tabular}{|c|c|c|c|c|c|c|}
\hline Model & OE & $\mathrm{NE}<5(\%)$ & $\mathrm{NE}<10(\%)$ & $\mathrm{NE}<15(\%)$ & $\mathrm{NE}<20(\%)$ & AIC \\
\hline Zero order & 27.25 & 6.15 & 13.85 & 41.54 & 60 & -13.46 \\
\hline First order & 25.35 & 10.77 & 43.08 & 61.54 & 66.15 & -19.01 \\
\hline Higuchi & 19.46 & 12.31 & 33.85 & 56.92 & 63.08 & -23.57 \\
\hline Hixson- Crowell & 23.58 & 9.23 & 35.38 & 58.46 & 66.15 & -19.55 \\
\hline Square rout of mass & 24.94 & 12.31 & 33.85 & 56.92 & 63.08 & -17.03 \\
\hline Three second rout of mass & 25.88 & 12.31 & 30.77 & 53.85 & 61.54 & -16.91 \\
\hline Weibull & 8.79 & 46.15 & 73.85 & 83.08 & 93.85 & -34.93 \\
\hline Korsmeyer- Peppas & 11.89 & 32.31 & 56.92 & 64.62 & 75.38 & -26.87 \\
\hline
\end{tabular}


Table 2: The values of E\%, AIC, coefficient of determination $\left(\mathrm{R}^{2}\right)$ and the regression parameters extracted from each data set was fitted in Weibull equation

\begin{tabular}{|c|c|c|c|c|c|c|c|c|}
\hline No & $\begin{array}{l}\text { Name of bioactive } \\
\text { molecule }\end{array}$ & $\begin{array}{c}\text { Nanoparticle } \\
\text { type }\end{array}$ & 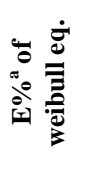 & 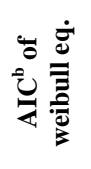 & 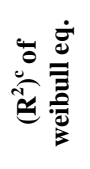 & 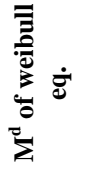 & 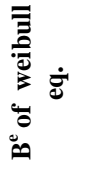 & Reff. $^{f}$ \\
\hline 1 & 5- fluorouracil & Chitosan & 1.02 & -75.63 & 0.998 & 0.31 & -0.44 & (Li et al., 2011) \\
\hline 2 & 5-Fluorouracil & $\begin{array}{l}\text { Chitosan coated sodium alginate- } \\
\text { chitosan }\end{array}$ & 2.12 & -54.66 & 0.996 & 0.67 & -1.11 & (Nagarwal et al., 2012) \\
\hline 3 & $\begin{array}{l}\text { All-trans retinoic acid } \\
\text { (ATRA) }\end{array}$ & $\begin{array}{l}\text { carboxymethylated }(\mathrm{CM}) \text {-curdlan with } \\
\text { a sulfonylurea }(\mathrm{SU})\end{array}$ & 5.55 & -59.31 & 0.982 & 0.59 & -2.86 & (Na et al., 2000) \\
\hline 4 & Amphotericin B & Gelatin & 3.75 & -43.75 & 0.891 & 0.39 & -1.3 & (Nahar et al., 2008) \\
\hline 5 & Artificial tear fluid & Chitosan-sodium alginate & 9.8 & -25.36 & 0.956 & 0.72 & -0.92 & (Motwani et al., 2008) \\
\hline 6 & Aspirin & Chitosan & 1.96 & -31.81 & 0.953 & 0.22 & -0.52 & (Shi et al., 2014) \\
\hline 7 & BMP-7/TGF-b2 & Alginate & 3.38 & -26.29 & 0.986 & 0.55 & -1.34 & (Patel and Nesamony, 2014) \\
\hline 8 & Brimonidine Tartrate & Eudragit & 11.2 & -36.25 & 0.935 & 0.66 & -1.89 & (Bhagav et al., 2011) \\
\hline 9 & Broadleaf holly leaf & Chitosan & 6.75 & -23.97 & 0.938 & 0.54 & -0.71 & (Zhang et al., 2015) \\
\hline 10 & $\begin{array}{l}\text { BSA as a model protein } \\
\text { drug }\end{array}$ & Chitosan & 7.86 & -14.39 & 0.836 & 0.34 & -0.48 & (Gan and Wang, 2007) \\
\hline 11 & $\begin{array}{l}\text { BSA as a model protein } \\
\text { drug }\end{array}$ & Poly(vinyl alcohol) & 2.71 & -53.49 & 0.997 & 0.77 & -1.42 & (Li et al., 1998) \\
\hline 12 & $\begin{array}{l}\text { BSA as a model protein } \\
\text { drug }\end{array}$ & Chitosan/alginate & 16.42 & -14.49 & 0.844 & 0.61 & -1.91 & (Li et al., 2007) \\
\hline 13 & $\begin{array}{l}\text { BSA as a model protein } \\
\text { drug }\end{array}$ & $\begin{array}{l}\text { Chitosan (L-aspartic } \quad \text { acid)- } \\
\text { polyethylene glycol }\end{array}$ & 2.28 & -47.07 & 0.96 & 0.3 & -1.3 & (Shu et al., 2009) \\
\hline 14 & Chloroquine phosphate & Gelatin & 6.62 & -13.11 & 0.952 & 0.47 & -1.35 & (Bajpai and Choubey, 2006) \\
\hline 15 & Cisplatin & Hyaluronic Acid & 18.96 & -23.62 & 0.924 & 0.93 & -2.85 & (Jeong et al., 2008) \\
\hline 16 & Curcumin & Alginate-Chitosan-Pluronic & 17.4 & -21.68 & 0.954 & 0.98 & -3.79 & (Das et al., 2010) \\
\hline 17 & Curcumin & 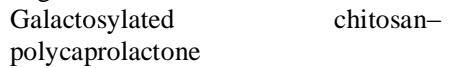 & 2.2 & -52.1 & 0.994 & 0.54 & -2.01 & (Zhou et al., 2013) \\
\hline 18 & Curcumin & $\begin{array}{l}\text { Chitosan-g-poly } \\
\text { isopropylacrylamide) }\end{array}$ & 19.89 & -16.85 & 0.965 & 1.19 & -3 & (Rejinold et al., 2011) \\
\hline 19 & Cyclosporin A & Chitosan & 1.67 & -33.03 & 0.7 & 0.04 & 0.05 & (De Campos et al., 2001) \\
\hline 20 & Diclofenac sodium & Eudragit ${ }^{\circledR}$ RS100 & 6.1 & -42.37 & 0.936 & 0.52 & 0.26 & (Barzegar-Jalali et al., 2012) \\
\hline 21 & Docetaxel & Chitosan & 3.67 & -49.35 & 0.989 & 0.53 & -3.23 & (Jain et al., 2014) \\
\hline 22 & Doxorubicine & $\begin{array}{l}\text { Acetylated hyaluronic } \quad \text { acid(low } \\
\text { molecular weight) }\end{array}$ & 3.35 & -53.11 & 0.985 & 0.46 & -1.48 & (Park et al., 2010) \\
\hline 23 & Doxycycline & Chitosan-gelatin & 2.55 & -28.22 & 0.983 & 0.67 & -1.26 & (Tormos et al., 2015) \\
\hline 24 & Essential oil & Alginate/cashew gum & 2.15 & -56.34 & 0.993 & 0.3 & -0.9 & (de Oliveira et al., 2014) \\
\hline 25 & FITC-BSA & Chondroitin sulfate-chitosan & 13.84 & -12.83 & 0.884 & 0.39 & -2.01 & (Yeh et al., 2011) \\
\hline 26 & FITC-BSA & Recombinant human gelatin & 9.15 & -60.51 & 0.969 & 1.02 & -3.15 & (Won and Kim, 2008) \\
\hline 27 & FITC-Dextran & Gelatin & 1.65 & -49.19 & 0.997 & 0.97 & -1.3 & (Gupta et al., 2004) \\
\hline 28 & FITC-dextran & Polyvinylpyrrolidone & 5.82 & -60.1 & 0.985 & 0.87 & -1.89 & (Bharali et al., 2003) \\
\hline 29 & Gentamicin & Chitosan & 8.31 & -31.66 & 0.93 & 0.52 & -1.03 & (Ji et al., 2011) \\
\hline 30 & Heparin & Chitosan-hyaluronic acid & 6.52 & -8.09 & 0.989 & 1.12 & -2.19 & $\begin{array}{l}\text { (Oyarzun-Ampuero et al., } \\
\text { 2009) }\end{array}$ \\
\hline 31 & Insulin & Hyaluronic Acid & 47.89 & -9.34 & 0.861 & 1.63 & -8.56 & (Han et al., 2012) \\
\hline 32 & Insulin & Chitosan and Arabic gum & 8.75 & -29.66 & 0.975 & 0.64 & -3.36 & (Avadi et al., 2010) \\
\hline 33 & Insulin & Gelatin & 1.58 & -34.51 & 0.958 & 0.5 & -1.96 & (Goswami et al., 2009) \\
\hline 34 & Insulin & Alginate/Chitosan & 3.2 & -35.92 & 0.87 & 0.43 & -2.31 & (Sarmento et al., 2007) \\
\hline 35 & Insulin & Calcium Alginate & 3.36 & -24.35 & 0.949 & 0.2 & -0.27 & (Lim et al., 2010) \\
\hline 36 & Insulin & Chitosan & 0.93 & -32.13 & 0.976 & 0.21 & -0.85 & (Hecq et al., 2015) \\
\hline 37 & Insulin & $\begin{array}{l}\text { Alginate/Trimethyl Chitosan } \\
\text { nanoparticle Containing Cationic } \beta \text { - } \\
\text { Cyclodextrin Polymers }\end{array}$ & 51.1 & -14.85 & 0.866 & 1.67 & -8.89 & (Mansourpour et al., 2015) \\
\hline 38 & Insulin & Chitosan/alginate & 18.55 & -18.86 & 0.708 & 0.71 & -1.7 & (Mukhopadhyay et al., 2015) \\
\hline 39 & Ketoconazole & Chitosan & 4.67 & -48.48 & 0.994 & 1.04 & -5.43 & (Modi et al., 2013) \\
\hline 40 & Measles antigen & low molecularweight chitosan & 3.37 & -27.26 & 0.988 & 0.43 & -2.11 & (Biswas et al., 2015) \\
\hline 41 & Methotrexate & Gelatin & 4.45 & -56 & 0.994 & 0.93 & -2.83 & (Cascone et al., 2002) \\
\hline 42 & Methotrexate & Gelatin & 10.21 & -39.68 & 0.972 & 0.84 & -2.79 & (Cascone et al., 2002) \\
\hline 43 & Methotrexate & Gelatin & 13.76 & -34.98 & 0.958 & 0.79 & -2.89 & (Cascone et al., 2002) \\
\hline 44 & Methotrexate & chitosan & 17.97 & -30.98 & 0.937 & 0.81 & -2.61 & (Azadi et al., 2013) \\
\hline 45 & $\begin{array}{l}\text { Methylene blue as a drug } \\
\text { model }\end{array}$ & k-Carrageenan & 13.8 & -31.96 & 0.807 & 0.88 & -3.36 & (Daniel-da-Silva et al., 2011) \\
\hline 46 & Naproxen & Eudragit ${ }^{\circledR}$ RS100 & 29 & -22.61 & 0.822 & 0.7 & -1.31 & (Adibkia et al., 2011) \\
\hline 47 & Nile red (NR) & Eudragit & 0.56 & -19.52 & 0.75 & 0.26 & 1.41 & (Yoo et al., 2011) \\
\hline 48 & Nimodipine & Hyaluronan-methylcellulose & 5.51 & -18.4 & 0.889 & 0.4 & -0.69 & (Wang et al., 2009) \\
\hline 49 & Ovalbumin & Alginate coated chitosan & 17.3 & -18.55 & 0.821 & 0.44 & -2.56 & (Borges et al., 2006) \\
\hline 50 & $\begin{array}{l}\text { Ovalbumin as model } \\
\text { protein }\end{array}$ & Chitosan/Carrageenan & 26.7 & -22.71 & 0.834 & 1.21 & -3.14 & (Grenha et al., 2010) \\
\hline
\end{tabular}




\begin{tabular}{|c|c|c|c|c|c|c|c|c|}
\hline 51 & Paclitaxel & Gelatin & 1.38 & -39.02 & 0.979 & 0.37 & 0.42 & (Lu et al., 2004) \\
\hline 52 & Paclitaxel & Chitosan & 2.42 & -51.81 & 0.995 & 0.7 & -2.99 & (Majedi et al., 2014) \\
\hline 53 & Piroxicam & EudragitwRS100 & 3.6 & -50.62 & 0.992 & 0.59 & -0.55 & (Adibkia et al., 2007) \\
\hline 54 & Prednisone acetate & $\begin{array}{l}\text { Poly-r,â-[N-(2-hydroxyethyl)-L- } \\
\text { aspartamide]-g-poly(E-caprolactone) }\end{array}$ & 6.51 & -47.76 & 0.954 & 0.4 & -1.5 & (Miao et al., 2006) \\
\hline 55 & Propofol & Alginate & 4.09 & -72.58 & 0.979 & 0.39 & -1.39 & (Najafabadi et al., 2015) \\
\hline 56 & Rivastigmine & Chitosan & 6.16 & -33.23 & 0.989 & 0.57 & -3.27 & (Fazil et al., 2012) \\
\hline 57 & Silk peptide & Chitosan-poly(acrylic acid) & 8.16 & -43.71 & 0.894 & 0.68 & -2.73 & (Hu et al., 2002) \\
\hline 58 & Sulphamethoxazole & Gelatin & 2.12 & -25.04 & 0.991 & 0.73 & -2.84 & (Bajpai and Choubey, 2005) \\
\hline 59 & $\begin{array}{l}\text { Tetramethylrhodamine- } \\
\text { labeled dextran }\end{array}$ & Gelatin & 4.68 & -45.48 & 0.994 & 1.15 & -1.98 & (Kaul and Amiji, 2002) \\
\hline 60 & $\begin{array}{l}\text { Tetramethylrhodamine- } \\
\text { labeled dextran }\end{array}$ & Gelatin & 5.6 & -32.94 & 0.977 & 1.25 & -1.61 & (Kaul and Amiji, 2002) \\
\hline 61 & Timolol Maleate & Chitosan & 4.82 & -25.44 & 0.918 & 0.33 & -0.44 & $\begin{array}{l}\text { (Agnihotri and Aminabhavi, } \\
\text { 2007) }\end{array}$ \\
\hline 62 & Timolol maléate & Hyaluronic acid modified chitosan & 8.72 & -32.44 & 0.954 & 0.82 & -0.61 & (Wadhwa et al., 2010) \\
\hline 63 & Timolol maléate & Chitosan & 9.06 & -33.4 & 0.97 & 0.73 & -0.7 & (Wadhwa et al., 2010) \\
\hline 64 & Tizanidine hydrochloride & Gelatin & 13.91 & -33.09 & 0.893 & 0.77 & -1.75 & (Lee et al., 2012) \\
\hline 65 & $\begin{array}{l}\text { Vascular endothelial } \\
\text { growth factor (VEGF) }\end{array}$ & Hyaluronic acid/Chitosan & 2.84 & -14.72 & 0.78 & 0.26 & 0.53 & (Parajó et al., 2010) \\
\hline
\end{tabular}

Table 3: Residual sum square (RSS), total sum square (TSS) and residual minuse square (RMS) for each data sets.

\begin{tabular}{|c|c|c|c|c|c|c|}
\hline No & Name of bioactive molecule & Nanoparticle type & $\begin{array}{l}\text { RSS of } \\
\text { weibull } \\
\text { eq. }\end{array}$ & $\begin{array}{l}\text { TSS of } \\
\text { weibull } \\
\text { eq. }\end{array}$ & $\begin{array}{l}\text { RMS of } \\
\text { weibull } \\
\text { eq. }\end{array}$ & Ref. \\
\hline 1 & 5- fluorouracil & Chitosan & 0.004 & 2.01 & 0.0005 & (Li et al., 2011) \\
\hline 2 & 5-Fluorouracil & Chitosan coated sodium alginate-chitosan & 0.01 & 3.22 & 0.002 & (Nagarwal et al., 2012) \\
\hline 3 & All-trans retinoic acid (ATRA) & $\begin{array}{l}\text { carboxymethylated }(\mathrm{CM}) \text {-curdlan with a } \\
\text { sulfonylurea }(\mathrm{SU})\end{array}$ & 0.12 & 6.42 & 0.01 & (Na et al., 2000) \\
\hline 4 & Amphotericin B & Gelatin & 0.06 & 3.37 & 0.01 & (Nahar et al., 2008) \\
\hline 5 & Artificial tear fluid & Chitosan-sodium alginate & 0.49 & 11.25 & 0.06 & (Motwani et al., 2008) \\
\hline 6 & Aspirin & Chitosan & 0.01 & 0.21 & 0.002 & (Shi et al., 2014) \\
\hline 7 & BMP-7/TGF-b2 & Alginate & 0.03 & 1.95 & 0.01 & (Patel and Nesamony, 2014) \\
\hline 8 & Brimonidine Tartrate & Eudragit & 0.48 & 7.33 & 0.04 & (Bhagav et al., 2011) \\
\hline 9 & Broadleaf holly leaf & Chitosan & 0.24 & 3.86 & 0.034 & (Zhang et al., 2015) \\
\hline 10 & BSA as a model protein drug & Chitosan & 0.23 & 1.4 & 0.06 & (Gan and Wang, 2007) \\
\hline 11 & BSA as a model protein drug & Poly(vinyl alcohol) & 0.03 & 8.81 & 0.003 & (Li et al., 1998) \\
\hline 12 & BSA as a model protein drug & Chitosan/alginate & 0.99 & 6.4 & 0.14 & (Li et al., 2007) \\
\hline 13 & BSA as a model protein drug & $\begin{array}{l}\text { Chitosan (L-aspartic acid)-polyethylene } \\
\text { glycol }\end{array}$ & 0.04 & 1.16 & 0.005 & (Shu et al., 2009) \\
\hline 14 & Chloroquine phosphate & Gelatin & 0.03 & 0.67 & 0.01 & (Bajpai and Choubey, 2006) \\
\hline 15 & Cisplatin & Hyaluronic Acid & 1.19 & 15.68 & 0.13 & (Jeong et al., 2008) \\
\hline 16 & Curcumin & Alginate-Chitosan-Pluronic & 0.7 & 15.24 & 0.09 & (Das et al., 2010) \\
\hline 17 & Curcumin & Galactosylated chitosan-polycaprolactone & 0.03 & 4.51 & 0.003 & (Zhou et al., 2013) \\
\hline 18 & Curcumin & Chitosan-g-poly (N-isopropylacrylamide) & 0.46 & 13.08 & 0.11 & (Rejinold et al., 2011) \\
\hline 19 & Cyclosporin A & Chitosan & 0.01 & 0.03 & 0.002 & (De Campos et al., 2001) \\
\hline 20 & Diclofenac sodium & Eudragit@ RS100 & 0.76 & 11.82 & 0.07 & (Barzegar-Jalali et al., 2012) \\
\hline 21 & Docetaxel & Chitosan & 0.06 & 5.06 & 0.006 & (Jain et al., 2014) \\
\hline 22 & Doxorubicine & $\begin{array}{l}\text { Acetylated hyaluronic acid(low molecular } \\
\text { weight) }\end{array}$ & 0.07 & 4.67 & 0.01 & (Park et al., 2010) \\
\hline 23 & Doxycycline & Chitosan-gelatin & 0.1 & 5.81 & 0.02 & (Tormos et al., 2015) \\
\hline 24 & Essential oil & Alginate/cashew gum & 0.02 & 2.34 & 0.002 & (de Oliveira et al., 2014) \\
\hline 25 & FITC-BSA & Chondroitin sulfate-chitosan & 0.32 & 2.78 & 0.08 & (Yeh et al., 2011) \\
\hline 26 & FITC-BSA & Recombinant human gelatin & 0.67 & 21.53 & 0.03 & (Won and Kim, 2008) \\
\hline 27 & FITC-Dextran & Gelatin & 0.01 & 3.26 & 0.002 & (Gupta et al., 2004) \\
\hline 28 & FITC-dextran & Polyvinylpyrrolidone & 0.17 & 11.38 & 0.01 & (Bharali et al., 2003) \\
\hline 29 & Gentamicin & Chitosan & 0.62 & 8.72 & 0.05 & (Ji et al., 2011) \\
\hline 30 & Heparin & Chitosan-hyaluronic acid & 0.04 & 4.01 & 0.04 & $\begin{array}{l}\text { (Oyarzun-Ampuero et al., } \\
\text { 2009) }\end{array}$ \\
\hline 31 & Insulin & Hyaluronic Acid & 3.45 & 24.81 & 0.43 & (Han et al., 2012) \\
\hline 32 & Insulin & Chitosan and Arabic gum & 0.19 & 7.81 & 0.03 & (Avadi et al., 2010) \\
\hline 33 & Insulin & Gelatin & 0.03 & 0.73 & 0.01 & (Goswami et al., 2009) \\
\hline 34 & Insulin & Alginate/Chitosan & 0.03 & 0.26 & 0.01 & (Sarmento et al., 2007) \\
\hline 35 & Insulin & Calcium Alginate & 0.09 & 1.79 & 0.02 & (Lim et al., 2010) \\
\hline 36 & Insulin & Chitosan & 0.002 & 0.1 & 0.001 & (Hecq et al., 2015) \\
\hline 37 & Insulin & $\begin{array}{l}\text { Alginate/Trimethyl Chitosan nanoparticle } \\
\text { Containing Cationic } \beta \text {-Cyclodextrin } \\
\text { Polymers }\end{array}$ & 7.34 & 54.72 & 0.46 & (Mansourpour et al., 2015) \\
\hline 38 & Insulin & Chitosan/alginate & 4.35 & 14.92 & 0.2 & (Mukhopadhyay et al., 2015) \\
\hline 39 & Ketoconazole & Chitosan & 0.06 & 8.63 & 0.01 & (Modi et al., 2013) \\
\hline
\end{tabular}




\begin{tabular}{|c|c|c|c|c|c|c|}
\hline 40 & Measles antigen & low molecularweight chitosan & 0.02 & 1.81 & 0.01 & (Biswas et al., 2015) \\
\hline 41 & Methotrexate & Gelatin & 0.14 & 22.37 & 0.014 & (Cascone et al., 2002) \\
\hline 42 & Methotrexate & Gelatin & 0.74 & 25.77 & 0.07 & (Cascone et al., 2002) \\
\hline 43 & Methotrexate & Gelatin & 0.58 & 13.98 & 0.06 & (Cascone et al., 2002) \\
\hline 44 & Methotrexate & chitosan & 0.88 & 14.04 & 0.09 & (Azadi et al., 2013) \\
\hline 45 & Methylene blue as drug model & $\mathrm{k}$-Carrageenan & 1.36 & 4.81 & 0.1 & (Daniel-da-Silva et al., 2011) \\
\hline 46 & Naproxen & Eudragit ${ }^{\circledR}$ RS100 & 2.66 & 14.89 & 0.22 & (Adibkia et al., 2011) \\
\hline 47 & Nile red (NR) & Eudragit & 0.02 & 0.09 & 0.02 & (Yoo et al., 2011) \\
\hline 48 & Nimodipine & Hyaluronan-methylcellulose & 0.21 & 1.87 & 0.05 & (Wang et al., 2009) \\
\hline 49 & Ovalbumin & Alginate coated chitosan & 0.56 & 3.11 & 0.09 & (Borges et al., 2006) \\
\hline 50 & Ovalbumin as model protein & Chitosan/Carrageenan & 2.61 & 15.71 & 0.29 & (Grenha et al., 2010) \\
\hline 51 & Paclitaxel & Gelatin & 0.02 & 1.06 & 0.004 & (Lu et al., 2004) \\
\hline 52 & Paclitaxel & Chitosan & 0.04 & 8.75 & 0.005 & (Majedi et al., 2014) \\
\hline 53 & Piroxicam & EudragitwRS100 & 0.03 & 3.91 & 0.004 & (Adibkia et al., 2007) \\
\hline 54 & Prednisone acetate & $\begin{array}{l}\text { Poly-r,â-[N-(2-hydroxyethyl)-L- } \\
\text { aspartamide]-g-poly(E-caprolactone) }\end{array}$ & 0.3 & 6.51 & 0.02 & (Miao et al., 2006) \\
\hline 55 & Propofol & Alginate & 0.13 & 6.23 & 0.007 & (Najafabadi et al., 2015) \\
\hline 56 & Rivastigmine & Chitosan & 0.13 & 11.53 & 0.02 & (Fazil et al., 2012) \\
\hline 57 & Silk peptide & Chitosan-poly(acrylic acid) & 0.9 & 8.45 & 0.05 & (Hu et al., 2002) \\
\hline 58 & Sulphamethoxazole & Gelatin & 0.01 & 1.55 & 0.004 & (Bajpai and Choubey, 2005) \\
\hline 59 & $\begin{array}{l}\text { Tetramethylrhodamine-labeled } \\
\text { dextran }\end{array}$ & Gelatin & 0.06 & 10.03 & 0.01 & (Kaul and Amiji, 2002) \\
\hline 60 & $\begin{array}{l}\text { Tetramethylrhodamine-labeled } \\
\text { dextran }\end{array}$ & Gelatin & 0.27 & 11.95 & 0.03 & (Kaul and Amiji, 2002) \\
\hline 61 & Timolol Maleate & Chitosan & 0.07 & 0.86 & 0.01 & $\begin{array}{l}\text { (Agnihotri and Aminabhavi, } \\
\text { 2007) }\end{array}$ \\
\hline 62 & Timolol maléate & Hyaluronic acid modified chitosan & 0.05 & 14.19 & 0.08 & (Wadhwa et al., 2010) \\
\hline 63 & Timolol maléate & Chitosan & 0.33 & 11.04 & 0.04 & (Wadhwa et al., 2010) \\
\hline 64 & Tizanidine hydrochloride & Gelatin & 1.04 & 9.66 & 0.07 & (Lee et al., 2012) \\
\hline 65 & $\begin{array}{l}\text { Vascular endothelial growth } \\
\text { factor (VEGF) }\end{array}$ & Hyaluronic acid/Chitosan & 0.1 & 0.46 & 0.05 & (Parajó et al., 2010) \\
\hline
\end{tabular}

Table 4: The release exponent of Korsmeyer- Peppas equation for all data sets.

\begin{tabular}{|c|c|c|c|c|}
\hline No & Name of bioactive molecule & Nanoparticle type & $\begin{array}{c}\text { N of } \\
\text { Korsmeyer } \\
\text { Peppas } \\
\text { equation }\end{array}$ & Ref. \\
\hline 1 & 5- fluorouracil & Chitosan & 0.19 & (Li et al., 2011) \\
\hline 2 & 5-Fluorouracil & Chitosan coated sodium alginate-chitosan & 0.5 & (Nagarwal et al., 2012) \\
\hline 3 & All-trans retinoic acid (ATRA) & carboxymethylated (CM)-curdlan with a sulfonylurea (SU) & 0.46 & (Na et al., 2000) \\
\hline 4 & Amphotericin B & Gelatin & 0.24 & (Nahar et al., 2008) \\
\hline 5 & Artificial tear fluid & Chitosan-sodium alginate & 0.47 & (Motwani et al., 2008) \\
\hline 6 & Aspirin & Chitosan & 0.14 & (Shi et al., 2014) \\
\hline 7 & BMP-7/TGF-b2 & Alginate & 0.39 & (Patel and Nesamony, 2014) \\
\hline 8 & Brimonidine Tartrate & Eudragit & 0.44 & (Bhagav et al., 2011) \\
\hline 9 & Broadleaf holly leaf & Chitosan & 0.32 & (Zhang et al., 2015) \\
\hline 10 & BSA as a model protein drug & Chitosan & 0.19 & (Gan and Wang, 2007) \\
\hline 11 & BSA as a model protein drug & Poly(vinyl alcohol) & 0.48 & (Li et al., 1998) \\
\hline 12 & BSA as a model protein drug & Chitosan/alginate & 0.38 & (Li et al., 2007) \\
\hline 13 & BSA as a model protein drug & Chitosan (L-aspartic acid)-polyethylene glycol & 0.14 & (Shu et al., 2009) \\
\hline 14 & Chloroquine phosphate & Gelatin & 0.19 & (Bajpai and Choubey, 2006) \\
\hline 15 & Cisplatin & Hyaluronic Acid & 0.62 & (Jeong et al., 2008) \\
\hline 16 & Curcumin & Alginate-Chitosan-Pluronic & 0.81 & (Das et al., 2010) \\
\hline 17 & Curcumin & Galactosylated chitosan-polycaprolactone & 0.36 & (Zhou et al., 2013) \\
\hline 18 & Curcumin & Chitosan-g-poly (N-isopropylacrylamide) & 0.82 & (Rejinold et al., 2011) \\
\hline 19 & Cyclosporin A & Chitosan & 0.02 & (De Campos et al., 2001) \\
\hline 20 & Diclofenac sodium & Eudragit ${ }^{2}$ RS100 & 0.25 & (Barzegar-Jalali et al., 2012) \\
\hline 21 & Docetaxel & Chitosan & 0.38 & (Jain et al., 2014) \\
\hline 22 & Doxorubicine & Acetylated hyaluronic acid(low molecular weight) & 0.28 & (Park et al., 2010) \\
\hline 23 & Doxycycline & Chitosan-gelatin & 0.36 & (Tormos et al., 2015) \\
\hline 24 & Essential oil & Alginate/cashew gum & 0.2 & (de Oliveira et al., 2014) \\
\hline 25 & FITC-BSA & Chondroitin sulfate-chitosan & 0.31 & (Yeh et al., 2011) \\
\hline 26 & FITC-BSA & Recombinant human gelatin & 0.79 & (Won and Kim, 2008) \\
\hline 27 & FITC-Dextran & Gelatin & 0.61 & (Gupta et al., 2004) \\
\hline 28 & FITC-dextran & Polyvinylpyrrolidone & 0.67 & (Bharali et al., 2003) \\
\hline 29 & Gentamicin & Chitosan & 0.32 & (Ji et al., 2011) \\
\hline 30 & Heparin & Chitosan-hyaluronic acid & 0.86 & (Oyarzun-Ampuero et al., 2009) \\
\hline 31 & Insulin & Hyaluronic Acid & 1.35 & (Han et al., 2012) \\
\hline 32 & Insulin & Chitosan and Arabic gum & 0.48 & (Avadi et al., 2010) \\
\hline 33 & Insulin & Gelatin & 0.24 & (Goswami et al., 2009) \\
\hline 34 & Insulin & Alginate/Chitosan & 0.26 & (Sarmento et al., 2007) \\
\hline 35 & Insulin & Calcium Alginate & 0.2 & (Lim et al., 2010) \\
\hline
\end{tabular}




\begin{tabular}{|c|c|c|c|c|}
\hline 36 & Insulin & Chitosan & 0.12 & (Hecq et al., 2015) \\
\hline 37 & Insulin & $\begin{array}{l}\text { Alginate/Trimethyl Chitosan nanoparticle Containing Cationic } \\
\beta \text {-Cyclodextrin Polymers }\end{array}$ & 1.22 & (Mansourpour et al., 2015) \\
\hline 38 & Insulin & Chitosan/alginate & 0.48 & (Mukhopadhyay et al., 2015) \\
\hline 39 & Ketoconazole & Chitosan & 0.81 & (Modi et al., 2013) \\
\hline 40 & Measles antigen & low molecularweight chitosan & 0.33 & (Biswas et al., 2015) \\
\hline 41 & Methotrexate & Gelatin & 0.62 & (Cascone et al., 2002) \\
\hline 42 & Methotrexate & Gelatin & 0.62 & (Cascone et al., 2002) \\
\hline 43 & Methotrexate & Gelatin & 0.59 & (Cascone et al., 2002) \\
\hline 44 & Methotrexate & chitosan & 0.68 & (Azadi et al., 2013) \\
\hline 45 & Methylene blue as drug model & k-Carrageenan & 0.5 & (Daniel-da-Silva et al., 2011) \\
\hline 46 & Naproxen & Eudragit@ RS100 & 0.47 & (Adibkia et al., 2011) \\
\hline 47 & Nile red (NR) & Eudragit & 0.02 & (Yoo et al., 2011) \\
\hline 48 & Nimodipine & Hyaluronan-methylcellulose & 0.16 & (Wang et al., 2009) \\
\hline 49 & Ovalbumin & Alginate coated chitosan & 0.34 & (Borges et al., 2006) \\
\hline 50 & Ovalbumin as model protein & Chitosan/Carrageenan & 0.87 & (Grenha et al., 2010) \\
\hline 51 & Paclitaxel & Gelatin & 0.15 & (Lu et al., 2004) \\
\hline 52 & Paclitaxel & Chitosan & 0.44 & (Majedi et al., 2014) \\
\hline 53 & Piroxicam & EudragitwRS100 & 0.43 & (Adibkia et al., 2007) \\
\hline 54 & Prednisone acetate & $\begin{array}{l}\text { Poly-r,â-[N-(2-hydroxyethyl)-L-aspartamide]-g-poly(E- } \\
\text { caprolactone) }\end{array}$ & 0.25 & (Miao et al., 2006) \\
\hline 55 & Propofol & Alginate & 0.26 & (Najafabadi et al., 2015) \\
\hline 56 & Rivastigmine & Chitosan & 0.87 & (Fazil et al., 2012) \\
\hline 57 & Silk peptide & Chitosan-poly(acrylic acid) & 0.3 & (Hu et al., 2002) \\
\hline 58 & Sulphamethoxazole & Gelatin & 0.43 & (Bajpai and Choubey, 2005) \\
\hline 59 & $\begin{array}{l}\text { Tetramethylrhodamine-labeled } \\
\text { dextran }\end{array}$ & Gelatin & 0.92 & (Kaul and Amiji, 2002) \\
\hline 60 & $\begin{array}{l}\text { Tetramethylrhodamine-labeled } \\
\text { dextran }\end{array}$ & Gelatin & 0.85 & (Kaul and Amiji, 2002) \\
\hline 61 & Timolol Maleate & Chitosan & 0.19 & $\begin{array}{l}\text { (Agnihotri and Aminabhavi, } \\
\text { 2007) }\end{array}$ \\
\hline 62 & Timolol maléate & Hyaluronic acid modified chitosan & 0.44 & (Wadhwa et al., 2010) \\
\hline 63 & Timolol maléate & Chitosan & 0.43 & (Wadhwa et al., 2010) \\
\hline 64 & Tizanidine hydrochloride & Gelatin & 0.55 & (Lee et al., 2012) \\
\hline 65 & $\begin{array}{l}\text { Vascular endothelial growth } \\
\text { factor (VEGF) }\end{array}$ & Hyaluronic acid/Chitosan & 0.05 & (Parajó et al., 2010) \\
\hline
\end{tabular}

\section{CONCLUSIONS}

Since drug release kinetics provide important information into realizing and optimizing nanoparticle drug delivery systems, there are different methods for the determination of release kinetics from such formulations(Hamidi et al., 2013). In this study we try to provide a comparative mathematical analysis of drug release from swellable polymeric delivery systems to find a general model applicable to multi mechanistic release. Drug release data from various swellable polymeric nanoparticles extracted from literatures were applied to the eight conventional models. Based on results, Weibull model seems to describe the release process with the major applicability. These viewpoints endorse that Weibull model seems to be flexible enough to describe the effect of system properties on release process and be more applicable for identification of drug release from swellable polymeric nanoparticles leading to the desired release profile in vivo.

\section{Financial support and sponsorship: Nil.}

Conflict of Interests: There are no conflicts of interest.

\section{REFERENCES}

Adibkia K, Javadzadeh Y, Dastmalchi S, Mohammadi G, Niri FK and Alaei-Beirami M, Naproxen-eudragit ${ }^{\circledR}$ rs 100 nanoparticles:
Preparation and physicochemical characterization. Colloids Surf B Biointerfaces. 2011, 83(1): 155-9.

Adibkia K, Shadbad MRS, Nokhodchi A, Javadzedeh A, Barzegar-Jalali M, Barar J, Mohammadi G and Omidi Y, Piroxicam nanoparticles for ocular delivery: Physicochemical characterization and implementation in endotoxin-induced uveitis. J Drug Target. 2007, 15(6): 407-16.

Agnihotri SA and Aminabhavi TM, Chitosan nanoparticles for prolonged delivery of timolol maleate. Drug Dev Ind Pharm. 2007, 33(11): 1254-62.

Aksungur P, Demirbilek M, Denkbaş EB, Vandervoort J, Ludwig A and Ünlü N, Development and characterization of cyclosporine a loaded nanoparticles for ocular drug delivery: Cellular toxicity, uptake, and kinetic studies. J Control Release. 2011, 151(3): 286-94.

Ashrafi H, Amini M, Mohammadi-Samani S, Ghasemi Y, Azadi A, Tabandeh MR, Kamali-Sarvestani E and Daneshamouz S, Nanostructure 1-asparaginase-fatty acid bioconjugate: Synthesis, preformulation study and biological assessment. Int J Biol Macromol. 2013, 62: 180-7.

Ashrafi $\mathrm{H}$ and Azadi A, Chitosan-based hydrogel nanoparticle amazing behaviors during transmission electron microscopy. Int J Biol Macromol. 2016, 84: 31-4.

Avadi MR, Sadeghi AMM, Mohammadpour N, Abedin S, Atyabi F, Dinarvand R and Rafiee-Tehrani M, Preparation and characterization of insulin nanoparticles using chitosan and arabic gum with ionic gelation method. Nanomedicine. 2010, 6(1): 58-63.

Azadi A and Ashrafi H, Cell organelle-shaped liposomes: A novel approach to present the stable intracellular drug delivery systems. Trends in Pharmaceutical Sciences. 2016, 2(2).

Azadi A, Hamidi M, Khoshayand M-R, Amini M and Rouini M-R, Preparation and optimization of surface-treated methotrexate-loaded nanogels intended for brain delivery. Carbohydr Polym. 2012, 90(1): 46271. 
Azadi A, Hamidi M and Rouini M-R, Methotrexate-loaded chitosan nanogels as 'trojan horses' for drug delivery to brain: Preparation and in vitro/in vivo characterization. Int J Biol Macromol. 2013, 62: 52330 .

Azadi A, Rouini M-R and Hamidi M, Neuropharmacokinetic evaluation of methotrexate-loaded chitosan nanogels. Int J Biol Macromol. 2015, 79: 326-35.

Bajpai A and Choubey J, Design of gelatin nanoparticles as swelling controlled delivery system for chloroquine phosphate. J Mater Sci Mater Med. 2006, 17(4): 345-58.

Bajpai A and Choubey J, Release study of sulphamethoxazole controlled by swelling of gelatin nanoparticles and drug-biopolymer interaction. J. Macromol. Sci., Pure Appl. Chem. 2005, 42(3): 253-75.

Barzegar-Jalali M, Adibkia K, Valizadeh H, Shadbad MRS, Nokhodchi A, Omidi Y, Mohammadi G, Nezhadi SH and Hasan M, Kinetic analysis of drug release from nanoparticles. J Pharm Pharm Sci. 2008, 11(1): 167-77.

Barzegar-Jalali M, Alaei-Beirami M, Javadzadeh Y, Mohammadi G, Hamidi A, Andalib S and Adibkia K, Comparison of physicochemical characteristics and drug release of diclofenac sodiumeudragit ${ }^{\circledR}$ rs100 nanoparticles and solid dispersions. Powder Technol. 2012, 219: 211-6.

Bei Y, Chen X, Liu Y, Xu J, Wang W, Gu Z, Xing K, Zhu A, Chen W and Shi L, Novel norcantharidin-loaded liver targeting chitosan nanoparticles to enhance intestinal absorption. Int J Nanomedicine. 2012, 7: $1819-27$

Bhagav P, Upadhyay $\mathrm{H}$ and Chandran S, Brimonidine tartrateeudragit long-acting nanoparticles: Formulation, optimization, in vitro and in vivo evaluation. AAPS PharmSciTech. 2011, 12(4): 1087-101.

Bharali DJ, Sahoo SK, Mozumdar S and Maitra A, Cross-linked polyvinylpyrrolidone nanoparticles: A potential carrier for hydrophilic drugs. J Colloid Interface Sci. 2003, 258(2): 415-23.

Biswas S, Chattopadhyay M, Sen KK and Saha MK, Development and characterization of alginate coated low molecular weight chitosan nanoparticles as new carriers for oral vaccine delivery in mice. Carbohydr Polym. 2015, 121: 403-10.

Borges O, Cordeiro-da-Silva A, Romeijn SG, Amidi M, de Sousa A, Borchard G and Junginger HE, Uptake studies in rat peyer's patches, cytotoxicity and release studies of alginate coated chitosan nanoparticles for mucosal vaccination. J Control Release. 2006, 114(3): $348-58$.

Cascone MG, Lazzeri L, Carmignani $\mathrm{C}$ and Zhu Z, Gelatin nanoparticles produced by a simple w/o emulsion as delivery system for methotrexate. J Mater Sci Mater Med. 2002, 13(5): 523-6.

Costa P and Lobo JMS, Modeling and comparison of dissolution profiles. Eur J Pharm Sci. 2001, 13(2): 123-33.

Daniel-da-Silva AL, Ferreira L, Gil AM and Trindade T, Synthesis and swelling behavior of temperature responsive $\kappa$-carrageenan nanogels. J Colloid Interface Sci. 2011, 355(2): 512-7.

Das RK, Kasoju N and Bora U, Encapsulation of curcumin in alginate-chitosan-pluronic composite nanoparticles for delivery to cancer cells. Nanomedicine. 2010, 6(1): 153-60.

Dash S, Murthy PN, Nath L and Chowdhury P, Kinetic modeling on drug release from controlled drug delivery systems. Acta Pol Pharm. 2010, 67(3): 217-23.

De Campos AM, Sánchez A and Alonso MaJ, Chitosan nanoparticles: A new vehicle for the improvement of the delivery of drugs to the ocular surface. Application to cyclosporin a. Int J Pharm. 2001, 224(1): 159-68.

de Oliveira EF, Paula HC and de Paula RC, Alginate/cashew gum nanoparticles for essential oil encapsulation. Colloids Surf B Biointerfaces. 2014, 113: 146-51.

Fazil M, Md S, Haque S, Kumar M, Baboota S, kaur Sahni J and Ali J, Development and evaluation of rivastigmine loaded chitosan nanoparticles for brain targeting. Eur J Pharm Sci. 2012, 47(1): 6-15.

Gan Q and Wang T, Chitosan nanoparticle as protein delivery carrier - systematic examination of fabrication conditions for efficient loading and release. Colloids Surf B Biointerfaces. 2007, 59(1): 24-34.
Goswami S, Bajpai J and Bajpai A, Designing gelatin nanocarriers as a swellable system for controlled release of insulin: An invitro kinetic study. J. Macromol. Sci., Pure Appl. Chem. 2009, 47(2): 11930

Grenha A, Gomes ME, Rodrigues M, Santo VE, Mano JF, Neves NM and Reis RL, Development of new chitosan/carrageenan nanoparticles for drug delivery applications. J Biomed Mater Res A. 2010, 92(4): 1265-72.

Gupta AK, Gupta M, Yarwood SJ and Curtis AS, Effect of cellular uptake of gelatin nanoparticles on adhesion, morphology and cytoskeleton organisation of human fibroblasts. J Control Release. 2004, 95(2): 197-207.

Hamidi M, Azadi A, Mohamadi-Samani S, Rafiei P and Ashrafi $\mathrm{H}$, Valproate-loaded hydrogel nanoparticles: Preparation and characterization. Journal of Applied Polymer Science. 2012, 124(6): 468693.

Hamidi M, Azadi A and Rafiei P, Hydrogel nanoparticles in drug delivery. Advanced drug delivery reviews. 2008, 60(15): 1638-49.

Hamidi M, Azadi A, Rafiei P and Ashrafi H, A pharmacokinetic overview of nanotechnology-based drug delivery systems: An admeoriented approach. Critical Reviews ${ }^{\mathrm{TM}}$ in Therapeutic Drug Carrier Systems. 2013, 30(5).

Hamidi M, Rafiei P, Azadi A and Mohammadi-Samani S, Encapsulation of valproate-loaded hydrogel nanoparticles in intact human erythrocytes: A novel nano-cell composite for drug delivery. J Pharm Sci. 2011, 100(5): 1702-11.

Han L, Zhao Y, Yin L, Li R, Liang Y, Huang H, Pan S, Wu C and Feng $\mathrm{M}$, Insulin-loaded ph-sensitive hyaluronic acid nanoparticles enhance transcellular delivery. AAPS PharmSciTech. 2012, 13(3): 836-45.

Hecq J, Siepmann F, Siepmann J, Amighi K and Goole J, Development and evaluation of chitosan and chitosan derivative nanoparticles containing insulin for oral administration. Drug Dev Ind Pharm. 2015;(ahead-of-print): 1-8

Hu Y, Jiang X, Ding Y, Ge H, Yuan Y and Yang C, Synthesis and characterization of chitosan-poly (acrylic acid) nanoparticles. Biomaterials. 2002, 23(15): 3193-201.

Jafari-Aghdam N, Adibkia K, Payab S, Barzegar-Jalali M, Parvizpur A, Mohammadi G and Sabzevari A, Methylprednisolone acetate-eudragit ${ }^{\circledR}$ rs100 electrospuns: Preparation and physicochemical characterization. Artificial cells, nanomedicine, and biotechnology. 2016 , 44(2): 497-503.

Jain A, Thakur K, Kush P and Jain UK, Docetaxel loaded chitosan nanoparticles: Formulation, characterization and cytotoxicity studies. Int J Biol Macromol. 2014, 69: 546-53.

Jeong YI, Kim ST, Jin SG, Ryu HH, Jin YH, Jung TY, Kim IY and Jung S, Cisplatin-incorporated hyaluronic acid nanoparticles based on ion-complex formation. J Pharm Sci. 2008, 97(3): 1268-76.

$\mathrm{Ji}$ J, Hao S, Wu D, Huang $\mathrm{R}$ and $\mathrm{Xu} \mathrm{Y}$, Preparation, characterization and in vitro release of chitosan nanoparticles loaded with gentamicin and salicylic acid. Carbohydr Polym. 2011, 85(4): 803-8.

Kalam MA, Humayun M, Parvez N, Yadav S, Garg A, Amin S, Sultana $\mathrm{Y}$ and Ali A, Release kinetics of modified pharmaceutical dosage forms: A review. Cont J Pharm Sci. 2007, 1: 30-5.

Kaul G and Amiji M, Long-circulating poly (ethylene glycol)modified gelatin nanoparticles for intracellular delivery. Pharm Res. 2002, 19(7): 1061-7.

Kosmidis K, Argyrakis P and Macheras P, A reappraisal of drug release laws using monte carlo simulations: The prevalence of the weibull function. Pharm Res. 2003, 20(7): 988-95.

Kumari A, Yadav SK and Yadav SC, Biodegradable polymeric nanoparticles based drug delivery systems. Colloids Surf B Biointerfaces. 2010, 75(1): 1-18

Lee EJ, Khan SA, Park JK and Lim K-H, Studies on the characteristics of drug-loaded gelatin nanoparticles prepared by nanoprecipitation. Bioprocess Biosyst Eng. 2012, 35(1-2): 297-307.

Li JK, Wang $\mathrm{N}$ and Wu XS, Poly (vinyl alcohol) nanoparticles prepared by freezing-thawing process for protein/peptide drug delivery. J Control Release. 1998, 56(1): 117-26. 
Li P, Wang Y, Peng Z, She F and Kong L, Development of chitosan nanoparticles as drug delivery systems for 5-fluorouracil and leucovorin blends. Carbohydr Polym. 2011, 85(3): 698-704.

Li T, Shi XW, Du YM and Tang YF, Quaternized chitosan/alginate nanoparticles for protein delivery. J Biomed Mater Res A. $2007,83(2): 383-90$.

Lim SM, Oh SH, Lee HH, Yuk SH, Im GI and Lee JH, Dual growth factor-releasing nanoparticle/hydrogel system for cartilage tissue engineering. J Mater Sci Mater Med. 2010, 21(9): 2593-600.

Liu $\mathrm{C}, \mathrm{Wu} \mathrm{Y}$, Zhao $\mathrm{L}$ and Huang $\mathrm{X}$, Preparation of acetylsalicylic acid-acylated chitosan as a novel polymeric drug for drug controlled release. Int J Biol Macromol. 2015, 78: 189-94.

Lu Z, Yeh T-K, Tsai M, Au JL-S and Wientjes MG, Paclitaxelloaded gelatin nanoparticles for intravesical bladder cancer therapy. Clin Cancer Res. 2004, 10(22): 7677-84.

Majedi FS, Hasani-Sadrabadi MM, VanDersarl JJ, Mokarram N, Hojjati-Emami S, Dashtimoghadam E, Bonakdar S, Shokrgozar MA, Bertsch A and Renaud P, On-chip fabrication of paclitaxel-loaded chitosan nanoparticles for cancer therapeutics. Adv Funct Mater. 2014, 24(4): 432 41.

Mansourpour M, Mahjub R, Amini M, Ostad SN, Shamsa ES, Rafiee-Tehrani $M$ and Dorkoosh FA, Development of acid-resistant alginate/trimethyl chitosan nanoparticles containing cationic $\beta$ cyclodextrin polymers for insulin oral delivery. AAPS PharmSciTech. 2015: 1-11.

Miao Z-M, Cheng S-X, Zhang X-Z and Zhuo R-X, Study on drug release behaviors of poly- $\alpha, \beta$-[n-(2-hydroxyethyl)-1-aspartamide]-gpoly ( $\varepsilon$-caprolactone) nano-and microparticles. Biomacromolecules. 2006, 7(6): 2020-6.

Modi J, Joshi G and Sawant K, Chitosan based mucoadhesive nanoparticles of ketoconazole for bioavailability enhancement: Formulation, optimization, in vitro and ex vivo evaluation. Drug Dev Ind Pharm. 2013, 39(4): 540-7.

Motwani SK, Chopra S, Talegaonkar S, Kohli K, Ahmad FJ and Khar RK, Chitosan-sodium alginate nanoparticles as submicroscopic reservoirs for ocular delivery: Formulation, optimisation and in vitro characterisation. Eur J Pharm Biopharm. 2008, 68(3): 513-25.

Mukhopadhyay P, Chakraborty S, Bhattacharya S, Mishra R and Kundu $\mathrm{P}, \mathrm{Ph}$-sensitive chitosan/alginate core-shell nanoparticles for efficient and safe oral insulin delivery. Int J Biol Macromol. 2015, 72: 640-8.

Na K, Park K-H, Kim SW and Bae YH, Self-assembled hydrogel nanoparticles from curdlan derivatives: Characterization, anticancer drug release and interaction with a hepatoma cell line (hepg2). J Control Release. 2000, 69(2): 225-36.

Nagarwal RC, Kumar R and Pandit J, Chitosan coated sodium alginate-chitosan nanoparticles loaded with 5-fu for ocular delivery: In vitro characterization and in vivo study in rabbit eye. Eur J Pharm Sci. 2012, 47(4): 678-85.

Nahar M, Mishra D, Dubey V and Jain NK, Development, characterization, and toxicity evaluation of amphotericin b-loaded gelatin nanoparticles. Nanomedicine. 2008, 4(3): 252-61.

Najafabadi AH, Azodi-Deilami S, Abdouss M, Payravand H and Farzaneh S, Synthesis and evaluation of hydroponically alginate nanoparticles as novel carrier for intravenous delivery of propofol. J Mater Sci Mater Med. 2015, 26(3): 1-11.

Oyarzun-Ampuero F, Brea J, Loza M, Torres D and Alonso M, Chitosan-hyaluronic acid nanoparticles loaded with heparin for the treatment of asthma. Int J Pharm. 2009, 381(2): 122-9.

Parajó Y, d'Angelo I, Welle A, Garcia-Fuentes M and Alonso MJ, Hyaluronic acid/chitosan nanoparticles as delivery vehicles for vegf and pdgf-bb. Drug deliv. 2010, 17(8): 596-604.

Park W, sub Kim K, Bae B-c, Kim Y-H and Na K, Cancer cell specific targeting of nanogels from acetylated hyaluronic acid with low molecular weight. Eur J Pharm Sci. 2010, 40(4): 367-75.
Patel K and Nesamony J, 2014. Preparation and characterization of insulin loaded calcium alginate nanoparticles. University of Toledo.

Rejinold NS, Sreerekha P, Chennazhi K, Nair S and Jayakumar $\mathrm{R}$, Biocompatible, biodegradable and thermo-sensitive chitosan-g-poly ( $\mathrm{n}$ isopropylacrylamide) nanocarrier for curcumin drug delivery. Int $\mathbf{J}$ Biol Macromol. 2011, 49(2): 161-72.

Sarmento B, Ribeiro A, Veiga F, Sampaio P, Neufeld R and Ferreira D, Alginate/chitosan nanoparticles are effective for oral insulin delivery. Pharm Res. 2007, 24(12): 2198-206.

Shi Y, Wan A, Shi Y, Zhang Y and Chen Y, Experimental and mathematical studies on the drug release properties of aspirin loaded chitosan nanoparticles. Biomed Res Int. 2014, 2014.

Shu S, Zhang X, Teng D, Wang Z and Li C, Polyelectrolyte nanoparticles based on water-soluble chitosan-poly (1-aspartic acid)polyethylene glycol for controlled protein release. Carbohydr Res. 2009, 344(10): 1197-204

Sun W, Zou Y, Guo Y, Wang L, Xiao X, Sun R and Zhao K, Construction and characterization of curcumin nanoparticles system. Journal of nanoparticle research. 2014, 16(3): 1-9.

Tormos CJ, Abraham C and Madihally SV, Improving the stability of chitosan-gelatin-based hydrogels for cell delivery using transglutaminase and controlled release of doxycycline. Drug Deliv Transl Res. 2015: 1-10.

van Boekel MA, On the use of the weibull model to describe thermal inactivation of microbial vegetative cells. International journal of food microbiology. 2002, 74(1): 139-59.

Wadhwa S, Paliwal R, Paliwal SR and Vyas S, Hyaluronic acid modified chitosan nanoparticles for effective management of glaucoma: Development, characterization, and evaluation. J Drug Target. 2010, 18(4): 292-302.

Wang Y, Lapitsky Y, Kang CE and Shoichet MS, Accelerated release of a sparingly soluble drug from an injectable hyaluronanmethylcellulose hydrogel. J Control Release. 2009, 140(3): 218-23.

Won Y-W and Kim Y-H, Recombinant human gelatin nanoparticles as a protein drug carrier. J Control Release. 2008, 127(2): 154-61.

Yang SC, Ge HX, Hu Y, Jiang XQ and Yang CZ, Doxorubicin loaded poly (butylcyanoacrylate) nanoparticles produced by emulsifier free emulsion polymerization. Journal of applied polymer science. 2000, 78(3): 517-26.

Yeh M-k, Cheng K-m, Hu C-s, Huang Y-c and Young J-j, Novel protein-loaded chondroitin sulfate-chitosan nanoparticles: Preparation and characterization. Acta Biomater. 2011, 7(10): 3804-12.

Yoo J-W, Giri $\mathrm{N}$ and Lee $\mathrm{CH}$, Ph-sensitive eudragit nanoparticles for mucosal drug delivery. Int J Pharm. 2011, 403(1): 262-7.

Yoon HY, Koo H, Choi KY, Kwon IC, Choi K, Park JH and Kim K, Photo-crosslinked hyaluronic acid nanoparticles with improved stability for in vivo tumor-targeted drug delivery. Biomaterials. 2013, 34(21): 5273-80.

Zhang H, Huang Q, Huang Z, Liu T and Li Y, Preparation and physicochemical properties of chitosan broadleaf holly leaf nanoparticles. Int J Pharm. 2015, 479(1): 212-8.

Zhou N, Zan X, Wang Z, Wu H, Yin D, Liao C and Wan Y, Galactosylated chitosan-polycaprolactone nanoparticles for hepatocytetargeted delivery of curcumin. Carbohydr Polym. 2013, 94(1): 420-9.

\section{How to cite this article:}

Azadi S, Ashrafi H, Azadi A, Mathematical modeling of drug release from swellable polymeric nanoparticles. J App Pharm Sci, 2017; 7 (04): 125-133. 